\title{
Maîtrise foncière dans les espaces ruraux. Un défi pour les projets de renaturation des cours d'eau
}

Land tenure: a challenge for river renaturation projects in rural areas

\section{Mathieu Bonnefond et Marie Fournier}

\section{(2) OpenEdition}

\section{Édition électronique}

URL : http://journals.openedition.org/economierurale/3908

DOI : 10.4000/economierurale.3908

ISSN : 2105-2581

\section{Éditeur}

Société Française d'Économie Rurale (SFER)

\section{Édition imprimée}

Date de publication : 15 mars 2013

Pagination : $55-68$

ISSN : 0013-0559

\section{Référence électronique}

Mathieu Bonnefond et Marie Fournier, « Maîtrise foncière dans les espaces ruraux. Un défi pour les projets de renaturation des cours d'eau », Économie rurale [En ligne], 334 | mars-avril 2013, mis en ligne le 15 mars 2015, consulté le 02 mai 2019. URL : http://journals.openedition.org/ economierurale/3908; DOI : 10.4000/economierurale.3908 


\title{
Maîtrise foncière dans les espaces ruraux Un défi pour les projets de renaturation des cours d'eau
}

\author{
Mathieu BONNEFOND, Marie FOURNIER • UMR 7324, Cités TERritoires Environnement Société
}

Dans cet article, les auteurs ont cherché à interroger les conditions de la mise en œuvre de l'action publique au sein des espaces ruraux et son impact sur les droits de propriété et sur les usages. L'étude des projets de restauration/renaturation menés dans le cadre du Contrat de rivière de la Veyle (Ain) fait ainsi apparaître que la maîtrise foncière est centrale et fait intégralement partie des stratégies développées par les acteurs publics en charge de la conduite de ces projets.

MOTS-CLÉS : action publique environnementale, espace rural, contrat de rivière, maîtrise foncière

\section{Land tenure: a challenge for river renaturation projects in rural areas}

In this article we aim at analyzing conditions of implementation of environmental public policy in rural areas and its impact on property rights and land uses. The study of renaturation projects on the Veyle river (France) reveals that land tenure is a central issue for the implementation of such projects and becomes a real part of the strategies developed by the public actors. (JEL: R5).

KEYWORDS: Environmental Policies, Rural Land, River Renaturation, Land Management

C et article interroge les conditions de mise en œuvre de l'action publique environnementale, plus particulièrement de celle menée dans le domaine de l'eau, au sein des espaces ruraux et leurs conséquences vis-à-vis des droits de propriété et d'usage.

Depuis quelques années, les injonctions se sont multipliées pour que les autorités en charge de la gestion et de l'entretien des cours d'eau engagent des projets de restauration ou de renaturation de leur linéaire, les cours d'eau étant des éléments structurants des activités et des territoires ruraux. Ces opérations trouvent leurs sources dans l'approche par bassin versant qui a accompagné le mouvement de décentralisation engagé au début des années 1980 (Ghiotti, 2007) et ont été confortées par les évolutions réglementaires survenues ces dernières années (Directive cadre sur l'eau, 2000 ; Loi sur l'eau et les milieux aquatiques, 2006). Les syndicats de rivières (le plus souvent des syndicats intercommunaux ou mixtes) sont devenus les relais privilégiés de cette politique qui se fonde sur une approche en termes de « milieu », définit les principes et caractéristiques du «bon fonctionnement » des hydrosystèmes et les érige en paradigme de l'action publique. Pour répondre à ces exigences, les opérateurs de la renaturation des cours d'eau s'appuient sur l'ingénierie écologique. À ce titre, si le terme de renaturation est souvent utilisé par commodité de langage (nous le faisons pour cet article) et comme traduction littérale du terme anglais renaturation, il renvoie aux opérations d'ingénierie écologique et, dans 
le cas qui nous préoccupe, aux opérations de restauration des cours d'eau'.

Or, dans le cadre de ces projets, la mâ̂trise foncière constitue un défi pour leurs porteurs. En effet, la mise en œuvre d'opérations de renaturation des cours d'eau, à la différence d'autres projets plus traditionnels d'ingénierie écologique, amène une spatialisation de l'action publique : ces dernières ne se cantonnent pas uniquement au lit mineur de la rivière mais impactent les espaces proches. De fait, elles créent des contraintes en matière de droits de propriété et d'usage sur ces derniers. Dans ces situations, il y a interaction entre politiques publiques et droits de propriété, c'est-àdire entre une action publique qui vise à résoudre un problème défini politiquement (en l'occurrence la qualité de l'eau et des milieux aquatiques) et un système d'allocation de ressources. Cette interaction conduit à la fois au développement par les acteurs publics de stratégies foncières et à la transformation des configurations locales des droits de propriété et d'usages (règles et détenteurs).

Cet article propose donc de s'interroger sur les conditions de la mise en œuvre de ces opérations sur une rivière non domaniale, c'est-à-dire soumise au régime de la propriété privée. Comment l'acteur public envisage-t-il le problème de la maîtrise foncière pour la mise en œuvre de projets de renaturation? Sous quelle forme passe cette maîtrise foncière ? Quelles sont les conséquences de l'action publique sur la configuration des droits de propriété et d'usage au sein des espaces impactés?

\footnotetext{
1. Dans ce cadre, il ne s'agit pas tant de favoriser le retour du cours d'eau à un état considéré comme «naturel» que de restaurer ses fonctions naturelles. En parlant de projets de renaturation des cours d'eau, nous retenons ici ceux inscrits dans une logique de « restauration active », où c'est par le biais d'aménagements et de travaux sur le cours d'eau que la restauration des fonctions naturelles de la rivière est engagée (Adam et al., 2007).
}

En nous focalisant sur les processus de mise en œuvre, nous souhaitons mettre en évidence la «phase pendant laquelle des actes et des effets sont générés à partir d'un cadre normatif d'intentions, de textes ou de discours : passer à l'acte, exécuter, gérer, administrer » (Mény et Thoenig, 1989). Nous mettrons l'accent sur la production de règles informelles et formelles liée à la maîtrise foncière dans la mise en œuvre de la politique de l'eau afin de faire ressortir les interactions multiples entre des acteurs publics et privés. En effet, la maîtrise de la propriété foncière constitue un lieu privilégié d'interactions entre les sphères publiques et privées. D'autre part, avec l'éclatement des centres de pouvoir, la place des acteurs privés dans l'action publique a évolué : de simples destinataires, ils peuvent dans certains cas devenir de véritables opérateurs des politiques publiques (Commaille et Duran, 2009). Ce changement implique des impératifs de régulation qui ne s'accommodent plus d'une simple logique d'arrangements informels (Duran et Thoenig, 1996 ; Commaille et Duran, 2009), mais nécessite la production de règles formelles négociées. Dans cet article, nous appréhenderons les processus de négociation comme la production d'ordres locaux (Crozier et Friedberg, 1977 ; Friedberg, 1993) pour le traitement à la fois des situations de tensions et celles inhérentes à l'organisation des rapports sociaux (Allain, 2004). Il s'agit bien de montrer les dimensions politiques et sociales de la production de ces règles secondaires de mise en œuvre de l'action publique environnementale (Larrue, 2000 ; Lascoumes, 1990 ; Lascoumes et Le Galès, 2007 ; Thoenig, 1998) et l'émergence de systèmes d'ordre locaux d'action publique (Lascoumes et Le Galès, 2007). Au-delà, l'analyse de la mise en œuvre des politiques publiques environnementales au niveau territorial montre qu'elles induisent des recompositions des modes de régulation mais aussi des territoires et des usages de ceux-ci. 
Pour illustrer notre propos, nous mobilisons l'exemple des projets de renaturation de cours d'eau menés sur le bassin de la Veyle (département de l'Ain) et rendons compte des recompositions et des ajustements qui ont eu lieu autour des opérations de maitrise de la ressource foncière dans ce cadre $^{2}$. La Veyle se situe à proximité de l'agglomération de Bourg-en-Bresse. Elle est à l'interface entre deux régions naturelles, la Bresse et la Dombes, auxquelles elle emprunte certaines caractéristiques : la partie amont du bassin versant correspond à la région d'étangs de la Dombes tandis que la partie aval correspond à une « rivière à moulins ", transformée et longée par des biefs et dérivations depuis plusieurs siècles. C'est un bassin versant fortement aménagé : dans sa partie aval, les dérivations de moulins et celles aménagées pour l'irrigation comptent pour près de $60 \mathrm{~km}$ de linéaire (Syndicat Mixte Veyle Vivante, 2004). En partie amont, un réseau séculaire de fossés a été progressivement construit pour permettre le ressuyage des terres et faciliter l'agriculture ; près de $1400 \mathrm{~km}$ de fossés ont été recensés. Sur le bassin versant, l'ensemble du linéaire des cours d'eau est sous le régime de la propriété privée. Le statut non domanial de l'ensemble du réseau hydrographique $\mathrm{du}$ bassin est pour notre propos particulièrement important. Pour rappel, les propriétaires des parcelles riveraines de chacun des cours d'eau possèdent également la rivière depuis ses berges et jusqu'à la moitié de son lit (article L215-2 du Code de l'environnement). Pour toute action

2. Cet article se fonde sur quelques-uns des résultats obtenus dans le cadre du projet de recherche New Rurality coordonné par le laboratoire CITERES et financé par l'Agence nationale de la recherche. Il se fonde sur le cadre conceptuel des régimes institutionnels (Knoepfel et al., 2001b) et l'analyse, menée dans trois pays (France, Pays-Bas, Suisse), se concentre sur des situations d'utilisation des ressources naturelles au sein des espaces ruraux. publique sur la rivière, notamment dans le cadre des opérations de renaturation, il est nécessaire de passer soit par des opérations de maîtrise foncière soit par des dispositifs juridiques permettant d'intervenir sur la propriété privée.

Les projets de renaturation menés sur le bassin versant de la Veyle étaient relativement précurseurs à l'échelon national ${ }^{3}$. Engagés au début des années 2000, ils s'inscrivaient dans le cadre d'un contrat de rivière, dont le porteur était le Syndicat Mixte Veyle Vivante ${ }^{4}$ Pour rappel, le contrat de rivière est un instrument d'intervention pensé à l'échelle des bassins versants ; il n'a pas de portée juridique mais constitue un engagement contractuel entre ses signataires (services de l'État, collectivités mais aussi potentiellement acteurs issus du monde socio-économique). Des objectifs de qualité des eaux, de gestion équilibrée des ressources et de valorisation des milieux aquatiques sont arrêtés afin d'adopter un programme d'interventions sur cinq ans ${ }^{5}$. Parmi ce programme d'interventions, plusieurs projets de renaturation étaient prévus. Or ces derniers ont nécessité des opérations de maîtrise foncière significatives.

Après un travail bibliographique exploratoire, c'est principalement par le biais d'entretiens semi-directifs tant avec le porteur des projets qu'avec les principaux usagers des espaces concernés (en particulier avec les différents acteurs socioéconomiques : agriculteurs, entreprise d'extraction de granulats) ainsi qu'avec le milieu associatif (association de pêche,

3. Si quelques projets de renaturation ont été engagés au cours des années 1990 (comme sur le bassin du Drugeon), ces derniers essaiment surtout depuis 2005 tant en milieu urbain que rural.

4. Nous utiliserons dans cet article les dénominations suivantes : « Syndicat de la Veyle » ou «SMVV » pour désigner à la fois l'institution, ses élus et ses techniciens.

5. www.eaufrance.fr, site consulté le 2 février 2012. 
association environnementaliste) que nous avons investi ce terrain.

Dans un premier temps, nous reviendrons donc sur les projets de renaturation qui ont amené le Syndicat de la Veyle à des opérations de maîtrise foncière sur des linéaires en propriété privée. Dans un second temps, nous montrerons que ce dernier a fait preuve à la fois d'un vrai pragmatisme et d'une stratégie à long terme pour l'acceptation locale de son action - privilégiant tant des processus souples et négociés que des procédures réglementaires fortes mais pouvant être génératrices de tensions, voire de conflits. Il apparaît alors que la mise en œuvre de l'action publique dans ce domaine ne passe pas forcément par la «publicisation » du linéaire des cours d'eau et que même lorsqu'elle a lieu, les gestionnaires donnent la préférence à des systèmes de conventionnement ou de contractualisation qui assurent le maintien d'activités au sein des espaces aménagés et par conséquent à la fois leur entretien et leur intégration territoriale.

\section{Des opérations de renaturation aux impacts fonciers importants}

Parmi ses différents volets, le Contrat de rivière de la Veyle signé en 2004 prévoyait un large panel de projets de renaturation sur le linéaire du bassin. Or la maîtrise foncière constituait pour leur porteur, le SMVV, un préalable à toute mise en œuvre.

\section{Des projets de renaturation " pilotes »}

Deux projets de renaturation d'ampleur étaient ainsi prévus : un projet de reméandrage sur la Veyle à proximité du lieu-dit du Moulin de Geai et celui de la création d'un nouveau lit pour la rivière sur

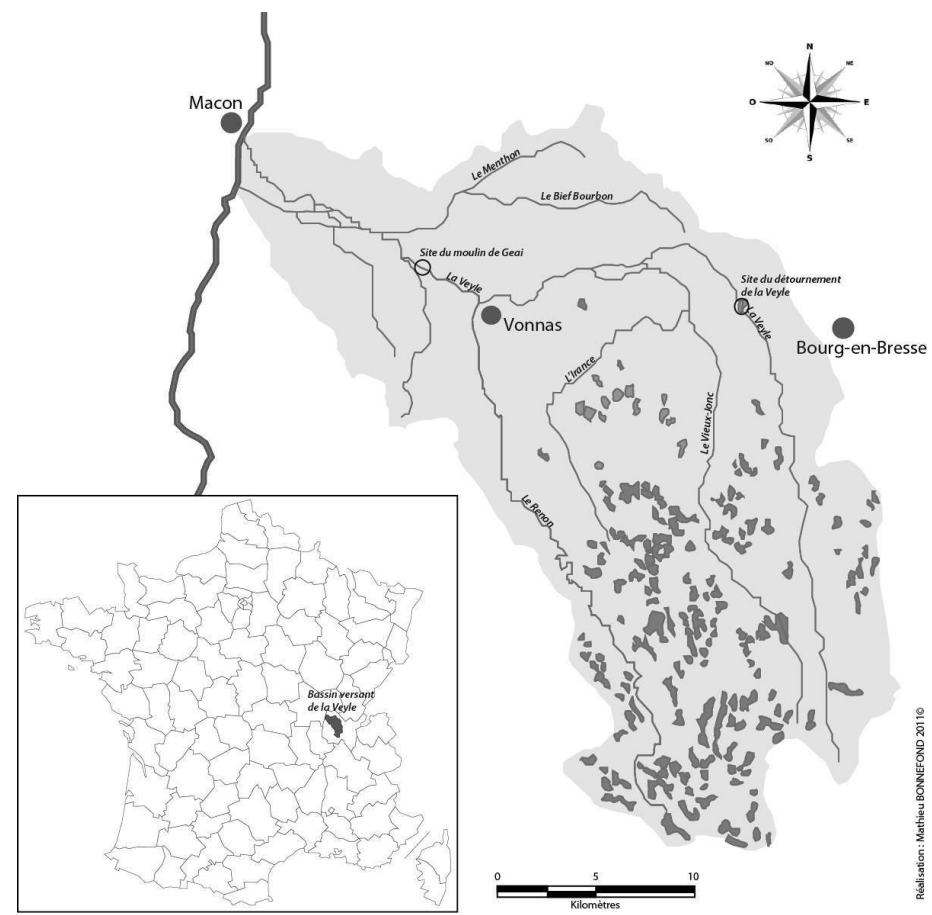

58 - Économie Rurale 334/Mars-Avril 2013 
RECHERCHE

quelques kilomètres (appelé le détournement de la Veyle). Dans cette région où les interventions et les contraintes sur les cours d'eau sont traditionnellement fortes, ces projets avaient été présentés comme étant des expérimentations, des initiatives de nature ponctuelle, non reproductibles et non généralisables, afin de limiter les tensions avec les différentes activités économiques dépendantes de la maîtrise de la ressource (agriculture).

Le premier site, situé sur la basse Veyle, au lieu-dit du Moulin de Geai (commune de Biziat), correspond à un ancien bief de moulin rectiligne, dont la ligne d'eau s'est fortement abaissée à la suite de la suppression du vannage, réduisant ainsi l'intérêt écologique du site. L'objectif était de redonner un caractère plus dynamique au lit en engageant une démarche pilote de reméandrage. Dans le cadre du Contrat de rivière, il s'agissait de la première opération de renaturation menée par le Syndicat de la Veyle. Pour ce dernier, l'ambition n'était pas tant de mener un projet techniquement exemplaire que de gagner en expérience d'un point de vue technique (techniques de restauration) mais aussi stratégique (identification des partenaires, mobilisation d'outils d'acquisition foncière, modalités de communication et sensibilisation). Dans la pratique, le SMVV a opté pour l'achat, à l'amiable, du foncier nécessaire à la réalisation du reméandrement. Le projet nécessitait une occupation foncière à la fois pour la réalisation des travaux et pour la nouvelle emprise de la rivière. Plus de $80 \%$ des terrains nécessaires à la réalisation des travaux prévus, soit une bande de trente mètres de large environ de chaque côté du tronçon de la petite Veyle concernée (sur un linéaire d'environ 500 mètres) ont été acquis. Cependant, trois propriétaires ont refusé de vendre leurs terrains en rive droite du cours d'eau (1,7 ha). Ces propriétaires, outre une opposition de principe, refusaient de perdre de la surface exploitable. Plusieurs possibilités avaient alors été identifiées par les techniciens du Syndicat de la Veyle pour pallier la non-maitrise foncière de ces terrains, depuis le statu quo (en évitant ces parcelles) à la Déclaration d'utilité publique (DUP), procédure longue et incertaine. Finalement, le projet ne constituant pas un enjeu majeur localement, les élus du Syndicat ont choisi la conciliation, se sont orientés vers une solution intermédiaire en évitant les parcelles non acquises par un nouveau tracé adapté et ont fait usage d'une Déclaration d'intérêt général (DIG) pour intervenir sur ces parcelles privées durant les travaux. Cette solution s'inscrit pour le SMVV dans une stratégie à plus long terme dans laquelle il s'agit de ne pas générer de conflits « durs » qui cristalliseraient les rapports entre les différentes parties prenantes ${ }^{6}$ et compromettraient par là même d'autres actions et notamment de futurs projets de renaturation.

Au sein du Syndicat de la Veyle, les enseignements tirés de ce premier projet ont servi lors de la mise en œuvre du second projet d'ampleur : le Détournement de la Veyle.

Depuis 1972, une exploitation de matériaux alluvionnaires autorisée s'est implantée dans le lit mineur de la Veyle, avec des impacts lourds : rupture du transport solide, décalage typologique des peuplements piscicoles, réchauffement de l'eau au niveau du passage dans la gravière, colmatage des fonds du lit de la Veyle. Dans les années 1990, des réflexions sont engagées par les élus locaux quant au déplacement du lit de la Veyle traversant le plan d'eau du site d'extraction. La Commune de Buellas, riveraine de l'exploitation de granulats, a révisé son Plan d'occupation des sols (POS)

6. Les projets de renaturation impliquent de nombreux acteurs, au-delà des propriétaires et usagers riverains directement visés : Chambre d'agriculture, élus locaux, services de l'État, associations, etc. Lors de nos enquêtes, le poids de ces derniers est apparu de façon prégnante, tant du fait des intérêts qu'ils défendent que des ressources qu'ils mobilisent dans ce cadre. 


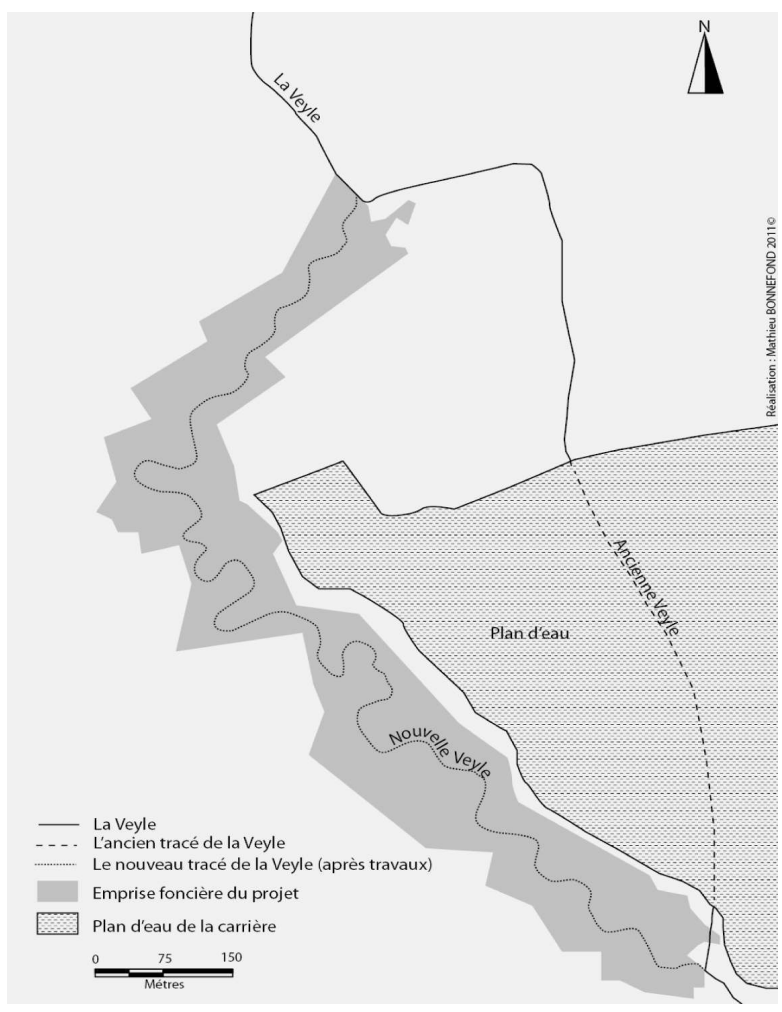

au milieu des années 1990 pour y inscrire un «espace réservé » dans l'optique d'utiliser ce périmètre pour réaménager un lit à la rivière. Il s'agissait pour les élus locaux d'affirmer une volonté politique forte visà-vis de leur cours d'eau. Lorsque le projet de contrat de rivière est lancé en 1999, le réaménagement du site est une évidence pour ses porteurs. L'idée est d'engager de lourds travaux de terrassement et d'aménager un nouveau lit mineur pour la Veyle sur un linéaire de près de $2 \mathrm{~km}$, avec la volonté de recréer un écosystème fonctionnel.

La réalisation de ce projet, inscrit au Contrat de rivière, nécessitait l'acquisition de plusieurs hectares de terres agricoles. Des négociations en ce sens ont été engagées dès le lancement du Contrat de rivière par le Syndicat de la Veyle. Cependant, ces dernières furent difficiles et la situation est restée bloquée plusieurs années jusqu'à ce que l'entreprise d'extraction, également signataire du Contrat de rivière, s'engage dans l'acquisition foncière au profit du SMVV. Finalement, près de dix hectares ont été achetés par ses soins, correspondant à l'emprise du projet. L'entreprise d'extraction a par la suite remis, au titre de l'euro symbolique, les terres au SMVV.

Les travaux de renaturation réalisés entre 2009 et 2010 ont consisté en la création d'un nouveau lit végétalisé et paysagé, découpé en trois tronçons distincts :

- un premier tronçon avec un tracé sinueux peu élaboré et des aménagements de berge légers,

- un second tronçon avec des méandres importants et des aménagements plus poussés, 
RECHERCHE

- un troisième tronçon avec des aménagements plus légers car potentiellement dynamique.

Les attendus sont une amélioration de la qualité de l'eau tant dans la rivière que dans l'étang d'extraction.

Ces opérations pilotes ne doivent pas faire oublier un certain nombre d'opérations de renaturation certes moins lourdes mais qui ont été menées sur tout le bassin.

\section{Des opérations ponctuelles de maîtrise foncière}

Le second groupe d'opérations à retenir correspond essentiellement à l'aménagement de frayères à brochets et à la restauration géomorphologique de petits tronçons.

L'aménagement des frayères à brochets implique une reconnexion de surface en prairie avec le cours d'eau afin de permettre la ponte et la maturation des jeunes brochets. Les différentes opérations conduites dans ce cadre ont nécessité l'acquisition de parcelles agricoles afin de pouvoir les inonder en période de reproduction. Du fait de cette inondabilité, ces dernières ne permettent plus que des pratiques agricoles extensives.

De plus, le contrat de rivière avait pour objectif la restauration éco-géomorphologique de tronçons dégradés au sein de secteurs encore fonctionnels par des interventions ciblées. Par la mise en place d'éléments physiques, l'aménagement de petits méandres artificiels ou l'installation de caches à poisson, l'ambition est de transformer le lit de rivière rectiligne et homogène et de restaurer les fonctions écologiques du cours d'eau pour qu'il soit plus favorable à la diversité de la faune piscicole. Dans cette même logique, des travaux d'entretien de la ripisylve ont également été menés, sur près d'un tiers du linéaire du bassin.
On rappellera enfin les projets de démantèlement des ouvrages en travers du lit mineur (vannages) n'ayant plus d'usage économique. À la suite du projet sur le Moulin de Geai, une étude a été lancée à l'échelle du bassin versant sur l'avenir des ouvrages présents (2006). Elle a abouti à l'identification de douze ouvrages appelés à être démantelés ou restaurés prioritairement. La plupart d'entre eux étant privés et rattachés à un droit d'eau, la question de leur acquisition ou du mode d'intervention sur la propriété privée reste posée à l'heure actuelle.

Ainsi, les opérations de renaturation présentées ci-dessus impliquent toutes une intervention relativement lourde sur les milieux, parfois avec des travaux de terrassement. Parallèlement à ces opérations, le Contrat de rivière de la Veyle prévoyait également l'utilisation de dispositifs pour contrôler ou interdire l'anthropisation des zones humides périphériques aux cours d'eau et préserver leurs dynamiques fluviales.

\section{Un espace de liberté surveillé Garantir des surfaces pour l'érosion naturelle}

Dans le cadre de son Contrat de rivière, le Syndicat de la Veyle avait également comme ambition de préserver des milieux de qualité. Pour ce faire, il propose la définition d'un « espace de liberté surveillé » sur l'Irance, un affluent de la Veyle, entre l'Illon et le Moulin du Loup. Il s'agit d'un secteur où la rivière conserve une dynamique fluviale active et un milieu écologique de qualité. Cette dynamique produit des méandres marqués et évolutifs dans le temps, avec une érosion naturelle des terrains riverains. L'expression « espace de liberté surveillé » est censée répondre à la fois aux objectifs de maintien de la dynamique fluviale et aux inquiétudes grandissantes localement quant au principe de «non-protection » des berges contre l'érosion. 
Pour ce projet, l'ambition du Syndicat de la Veyle était de passer par l'acquisition d'un linéaire important pour s'assurer qu'aucun aménagement ne viendrait remettre en question les dynamiques naturelles. À la suite des négociations avec les propriétaires et les exploitants locaux, le principe suivant a été arrêté : les parcelles à proximité du cours d'eau feraient l'objet d'une acquisition par le Syndicat de la Veyle, dans le cadre du Contrat de rivière, si le propriétaire en émettait le désir. De plus, il a été affiché comme principe que si un bâtiment était menacé par l'érosion, une protection pourrait être mise en place par le Syndicat.

En conclusion, ces exemples montrent comment les acteurs publics répondent aux impératifs législatifs de gestion des cours d'eau dans la mise en œuvre de leurs projets, par la production de règles négociées, par des ajustements stratégiques et par la mobilisation de ressources. Se pose en outre, à chaque fois, la question de la maîtrise foncière, car quasiment tous ont des impacts fonciers non négligeables. Nous verrons ainsi dans une seconde partie les moyens mobilisés pour aboutir à cette maîtrise foncière et les problèmes qu'elle soulève.

\section{La maîtrise foncière comme préalable à l'action publique environnementale}

Dans le cadre des opérations de maîtrise foncière, nous montrerons dans cette seconde partie que le Syndicat de la Veyle a dû développer différentes stratégies de négociation et de partenariat, tant avec les propriétaires fonciers et usagers de l'espace qu'avec les acteurs institutionnels (Chambre d'agriculture, Fédération de pêche et de protection des milieux aquatiques, etc.). Ces stratégies ont conduit à la formalisation de règles du jeu explicites et au développement de procédures contractuelles.

En outre, nous verrons que le Syndicat, confronté à la contingence de la question foncière, a dû développer une approche transversale, dépassant le simple traitement technique et appréhendant les projets dans leur dimension territoriale. Force est de constater que ces opérations sur le foncier ont produit des changements en matière d'utilisation de l'espace, de répartition des droits d'usage et d'organisation institutionnelle.

\section{Pragmatisme et recherche de l'acceptation locale face à la maîtrise foncière}

En observant les pratiques du Syndicat de la Veyle, il apparaît que ce dernier, pour arriver à ses objectifs, a fait preuve d'un vrai pragmatisme en mobilisant tant des procédures ad hoc portant sur les droits de propriété que d'autres formes d'action plus informelles.

Un syndicat de rivière aux prises avec un parcellaire privé et fortement investi Lorsqu'un projet est engagé sur un cours d'eau non domanial, son porteur se trouve rapidement confronté (en particulier dans le milieu rural) à un parcellaire fortement investi et privatisé, sur lequel différents droits d'usages se superposent ou se juxtaposent. Le droit de propriété constitue le support juridique de différents types de droits d'usages qui peuvent faire l'objet de transfert via des baux (baux ruraux, baux de pêche, baux de chasse...) mais il existe aussi d'autres formes contractuelles plus souples telles que les conventions d'échanges de parcelles entre exploitants. Dans le cas de notre étude, il apparaît que ces différentes formes contractuelles ou conventionnelles d'usage de l'espace s'imposent d'ailleurs parfois plus encore que le 
droit de propriété. Ainsi, lors de la mise en place du projet de détournement de la Veyle, le SMVV a d'abord conduit ses négociations pour l'acquisition de terrains avec les détenteurs des baux ruraux avant d'entamer les négociations avec leurs propriétaires. Ces derniers accordent en effet habituellement leur position sur celle de leurs fermiers, qui constituent la principale forme de valorisation de leur fonds. Les exploitants agricoles sont les premiers à avoir été au cœur des négociations quant au périmètre des parcelles à acquérir, à leur prix d'achat et aux indemnités d'éviction. Finalement, entre l'achat du terrain et les indemnités d'éviction, comme le souligne une des personnes enquêtées, les «parcelles sont achetées deux fois ", dans le sens où deux procédures de négociation sont nécessaires pour l'acquisition d'une même parcelle. Ceci montre la prédominance des ayants-droit, principalement les exploitants agricoles en fermage en milieu rural.

\section{Éviter les situations conflictuelles pour assurer une bonne mise en æuvre des projets}

Face à cette complexité et ces multiples contraintes qui caractérisent le parcellaire, il nous est d'abord apparu que les gestionnaires des cours d'eau ont tendance à adopter la stratégie de l'évitement, soit pour s'écarter des conflits pouvant s'étendre audelà du périmètre du projet et entraîner des blocages dans la mise en œuvre d'autres projets, soit tout simplement par économie de temps.

Par exemple, le SMVV a de préférence retenu des parcelles publiques, appartenant à ses communes membres, pour l'aménagement des frayères à brochets prévues dans le cadre du Contrat de rivière. Concrètement, les porteurs du contrat se sont voulus relativement pragmatiques et opportunistes pour ces projets. N'ayant pas identifié de sites au préalable à la signature du contrat, ils ont préféré fonctionner selon les opportunités foncières qui s'offraient à eux, après une première identification des sites potentiels (prairies inondées) par photos aériennes. Ce point est intéressant car il nous montre que dans le choix d'un site, la contrainte foncière devient un critère aussi légitime que ceux plus « objectifs » basés sur la qualité physique et hydraulique du site en question.

On retrouve une autre situation d'évitement dans le cadre du projet de constitution d'un « espace de liberté » prévu par le SMVV sur l'Irance. Les achats de parcelles dans ce périmètre y ont finalement été limités et ponctuels - non pas étendus et systématiques comme cela avait été envisagé au départ par certains élus et techniciens de la structure. Il s'agissait pourtant de l'un des derniers secteurs où la rivière conservait une forte dynamique géomorphologique et une volonté forte s'était exprimée au sein du Syndicat pour le préserver. Une démarche d'acquisition avait donc été engagée. Cette dernière a cependant rencontré de nombreuses réactions négatives de la part des propriétaires privés. Ces derniers ont pour la plupart refusé de céder leur terrain et ont également récusé le principe de «non-intervention » que souhaitait tenir le Syndicat de la Veyle face à l'érosion des berges. De plus, il était difficile pour le SMVV de justifier ces achats puisqu'il ne proposait aucun projet de travaux sur le site. Finalement, le parti pris a été d'abandonner la démarche d'achat systématique. Plusieurs autres raisons ont amené les SMVV à prendre cette décision : la question des ressources financières nécessaires à ce projet, ainsi que le problème de la gestion des surfaces acquises, le Syndicat n'ayant pas pour vocation à être un gestionnaire de foncier agricole. Cependant, en abandonnant ce projet, nos interlocuteurs au sein du SMVV reconnaissent que c'est le maintien, à terme, de cet « espace de liberté » qui est en question. 
Un syndicat qui passe par les achats amiables et les procédures ad hoc

$\mathrm{Si}$ le recours au foncier public constitue une stratégie souvent utilisée, certaines opérations nécessitent néanmoins pour le porteur des travaux prévus de disposer du foncier et donc des droits d'usage permanent ou temporaire sur des parcelles privées. Or, dans le cas que nous avons ici retenu, il apparaît que le Syndicat de la Veyle a souvent cherché à éviter les procédures réglementaires lourdes ayant pourtant ce type de vocation au départ telles que la Déclaration d'Utilité Publique (DUP) ou la Déclaration d'Intérêt Général (DIG) - pour leur préférer des procédures plus souples de négociation foncière. De même, si la DUP ou la DIG sont mobilisées ou évoquées par le Syndicat de la Veyle, ce n'est pas tant pour réaliser des expropriations ou intervenir « en force » sur la propriété privée que pour faciliter les négociations foncières et la réalisation des travaux. Ces procédures sont alors plus utilisées comme moyen de pression dans le processus de négociation pour inciter les propriétaires et les exploitants agricoles à entrer dans un processus de négociation sur le prix d'achat et non plus questionner son éventualité.

Si l'on reprend à nouveau l'exemple du projet de Détournement de la Veyle, le SMVV a d'abord cherché à acquérir à l'amiable les parcelles nécessaires au projet. Cependant, le contexte local était difficile : les propriétaires et les exploitations agricoles de la zone étaient à la fois peu favorables au projet et ne souhaitaient pas céder des terres cultivées à proximité de leur exploitation. De plus, la proximité avec le site d'extraction laissait entendre que la négociation du prix des terrains et des indemnités d'éviction se ferait sur cette base et non sur celle des terres agricoles. Bien que le Syndicat fût prêt à payer un prix supérieur à celui de la terre agricole, les propriétaires et exploitants se refusaient à vendre. Devant le retard que prenait le projet, une double démarche fut engagée. Celle-ci consistait d'une part à brandir la procédure de DUP, telle une menace face aux oppositions, tandis que d'autre part, l'entreprise d'extraction voisine était mobilisée dans la négociation foncière. Le Syndicat ne pouvait pas payer le foncier au prix des terres destinées à l'extraction (plus de dix fois supérieur au prix de la terre agricole), sans faire naître le risque de créer un précédent et de générer localement une inflation sur les terres agricoles. L'engagement de l'entreprise d'extraction dans la négociation foncière (non prévu dans le contrat de rivière) s'explique principalement par le fait que le renouvellement de son autorisation d'exploitation était conditionné par la réalisation du projet de détournement. Ce conditionnement avait été demandé par les élus locaux au préfet et a eu un effet majeur. Bien que ressenti comme une forme de chantage par l'entreprise d'extraction, cette dernière a repris à son compte les négociations avec les propriétaires et exploitants, et a acheté le parcellaire nécessaire au projet (près de dix hectares) à un prix médian entre ce que le Syndicat de la Veyle pouvait proposer et celui attendu par leurs propriétaires. À terme, l'entreprise d'extraction devait remettre à titre gracieux le parcellaire acquis au SMVV.

Le recours à la procédure de DUP avait déjà été envisagé par le SMVV dans le cadre du projet sur le Moulin du Geai pour finaliser l'acquisition foncière. Cependant, comme nous l'avons rappelé en première partie, le Syndicat de la Veyle avait finalement préféré revoir le tracé du reméandrage (au vu de la petite taille des parcelles non acquises) et passer par une Déclaration d'intérêt général (DIG) qui permettait d'intervenir de manière temporaire et légitime sur ces parcelles restées privées. Dans le cadre de ses interventions sur la ripisylve, dont l'entretien incombe 
aux propriétaires riverains (Art. L215-14 et suivant du Code de l'environnement), le Syndicat a également fait le choix de passer systématiquement par une DIG. La mobilisation de cette procédure permet de justifier la dépense publique réalisée sur des parcelles privées. Elle facilite et garantit le traitement efficace de linéaires importants, de manière cohérente. Cette procédure, maîtrisée par le Syndicat, lui permet à la fois d'agir dans un cadre réglementaire clair, stabilisé, et de ne pas cristalliser les oppositions. En effet, du fait de son caractère temporaire et ne portant pas atteinte, dans l'esprit des propriétaires à leurs droits sur le sol, cette dernière suscite peu de conflits.

Si notre premier constat a donc été celui d'une très grande souplesse et d'une véritable stratégie du Syndicat de la Veyle dans les dispositifs qu'il mobilise, il est également apparu que plus qu'une « publicisation » de l'espace, ces opérations de mâ̂trise foncière amènent à une recomposition des droits de propriété et d'usage, dans laquelle les premiers passent certes à un acteur public mais où l'usage reste privé.

\section{Maintien et recomposition des droits d'usage}

Dans le cadre des opérations de renaturation menées sur la Veyle et ses affluents, la préférence est souvent donnée par le SMVV à des formes de contractualisation ou de conventionnement plutôt qu'à l'achat. C'est ce qui a par exemple été tenté lors de la mise en place de frayères sur des parcelles privées ou communales. Plus encore, on retrouve ce schéma dans les opérations d'entretien de la ripisylve que le Syndicat a menées. Ce phénomène peut s'expliquer de deux manières. Les élus du Syndicat de la Veyle et son personnel technique nous rappellent ainsi que ce type de structure, qui n'est pas destinée à être pérenne sur une très longue durée, n'a nullement vocation à être à la tête d'un patrimoine foncier important ni n'est en mesure d'être contrainte par des baux ruraux. Lors de nos entretiens, l'ingénieur en charge de ces questions s'interroge sur l'entretien de ce foncier, ainsi que celle de son devenir et de son statut au moment de la dissolution du syndicat. Or, à l'inverse de l'achat, conventionnement et contractualisation garantissent à ce dernier le maintien d'une activité sur le site, en adéquation avec les exigences de l'entretien d'une zone réaménagée.

Ainsi, lorsque l'achat s'impose, le Syndicat de la Veyle s'est toujours attaché à maintenir des exploitants agricoles sur site. Le maintien d'une activité économique, sous conventionnement, constitue une garantie du bon entretien de l'aménagement. C'est le cas par exemple au Moulin de Geai, où un système de prêt à usage a été mis en place par le SMVV avec un agriculteur, ainsi que des accords informels pour l'entretien des berges. Dans le cas du Détournement de la Veyle, de multiples accords de partenariats ont été passés avec les usagers du site, tels que l'entreprise d'extraction, mais aussi l'association de pêche, les agriculteurs riverains, une association locale de protection de la nature...

Ce dernier point est également révélateur d'une évolution dans les pratiques des acteurs publics. L'action publique environnementale se nourrit de partenariats dans une logique d'efficacité et d'intégration territoriale (Commaille et Duran, 2009).

Vers une clarification et une simplification du parcellaire privé

Enfin, il nous est apparu que l'intervention publique, lors de ces opérations de maîtrise foncière, est également l'occasion d'une recomposition de l'utilisation de l'espace et des droits d'usage. C'est le cas par exemple au Moulin de Geai, où à la suite de la modification des parcelles, les exploitants agricoles ont établi une convention 
d'échanges entre eux afin de se redistribuer les terrains et de redonner une plus grande cohérence à leur parcellaire d'exploitation.

De la même manière, la procédure de DIG lancée pour l'entretien de la ripisylve a permis une clarification des baux de pêche sur le linéaire de la Veyle. Lorsque nous en avons parlé avec l'association agréée de pêche locale, ses membres nous ont ainsi expliqué que les droits de pêche s'appuyaient, avant la mise en place de cette procédure, sur des accords plus ou moins tacites et informels avec les propriétaires du linéaire. Le risque était donc toujours là de voir un jour une partie du cours de la rivière devenir impossible d'accès. Or, dans le cadre de la DIG établie pour l'entretien de la ripisylve, la possibilité a été offerte aux propriétaires d'éviter la contribution financière de $20 \%$ aux travaux et de céder en échange leur droit de pêche à l'association locale. Ces $20 \%$ sont en effet pris en charge par la Fédération départementale de pêche et de protection des milieux aquatiques. Cette solution a été le plus souvent retenue, ce qui a grandement facilité la réalisation des opérations d'entretien de la ripisylve et a permis de clarifier par là même la situation des baux de pêche sur des linéaires très importants.

$$
\begin{gathered}
* \\
* *
\end{gathered}
$$

Pour conclure cet article, nous voudrions tout d'abord revenir en quelques lignes sur nos principaux résultats, puis sur le contexte particulier dans lequel ils s'inscrivent, avant de souligner les questionnements plus généraux auxquels ils font écho.

Nous avons vu que les opérations de renaturation conduites dans le cadre du Contrat de rivière de la Veyle ont eu un impact foncier important, tant au niveau de leur emprise que dans la modification de la configuration des droits de propriété et d'usage localement. Dans ce cadre, le
Syndicat de la Veyle a été amené, à la fois par pragmatisme, par recherche de l'acceptation locale et par volonté d'intégration territoriale des projets, à mobiliser tant des procédures ayant spécifiquement cette vocation (DUP/DIG) que des procédures plus informelles et souples. Selon les contextes, le SMVV se trouve dans une situation où il a besoin de légitimer son action et de passer par des procédures reconnues auprès des acteurs et usagers locaux. Dans d'autres cas de figure, le recours à l'achat à l'amiable ou à des accords quant à l'utilisation de l'espace, plutôt qu'à des procédures d'expropriation, présente l'intérêt de limiter les crispations et les conflits. Un second point d'importance est que l'achat de foncier n'est pas la priorité (voire même préférerait être évité); lorsqu'il a lieu, des conventionnements ou contractualisations complémentaires sont recherchées pour garantir la permanence d'usages.

L'analyse menée au sein d'un espace rural, où l'activité agricole reste dominante, montre une déconnexion entre les droits d'usage et le droit de propriété. Il y a donc deux types d'interlocuteurs à appréhender pour l'acteur public : les propriétaires et les usagers. La prégnance des baux ruraux, liés à l'activité agricole, amène à ce que finalement les propriétaires jouent un rôle moindre par rapport aux exploitants. De même, le changement de propriétaire n'est pas l'enjeu principal, qui est alors plutôt celui du maintien des usages et des usagers. Nous avons vu cependant que le changement de propriété, en particulier l'achat par l'acteur public, amène à la disparition des baux ruraux et à l'émergence de nouvelles formes de droits d'usage.

Une autre spécificité des projets observés tient également à la nature des cours d'eau sur lesquels ils sont mis en ouvre. Ce sont en effet des cours d'eau non domaniaux, c'est-à-dire sur lesquels toute action publique nécessite une coopération avec les détenteurs privés des droits de propriété. 
RECHERCHE

Lors de notre étude de cas, nous avons vu que le Syndicat de la Veyle privilégie d'abord des projets pouvant être menés sur un parcellaire appartenant aux communes, comme par exemple dans l'aménagement de zones de frayères. Mais il nous semble ici particulièrement intéressant de montrer que l'action publique, pour atteindre ses objectifs, est, si ce n'est dépendante, du moins liée à la constitution de partenariats avec des acteurs non institutionnels.

Notre analyse de la mise en œuvre d'une politique publique environnementale au sein des espaces ruraux nous amène alors à identifier un certain nombre d'effets, qui s'expriment à la fois au niveau du droit, avec la recomposition des droits de propriété et d'usage, qu'au niveau du jeu d'acteurs, avec la construction de partenariats public/privé.

Les projets de renaturation produisent une recomposition de l'utilisation et de la répartition des droits d'usage de l'espace et des ressources naturelles, et entraînent également une nouvelle organisation locale. Les acteurs publics et privés sont conduits à négocier et à ajuster leurs stratégies. La mise en œuvre des projets de renaturation au sein d'espaces ruraux marqués par une prédominance de la propriété privée, tend à transformer deux types de relation :

- Les relations entre l'action publique et la propriété privée : les acteurs publics sont amenés à mobiliser des procédures relevant des droits de propriété dans le cadre de la mise en œuvre de l'action publique, modifiant ainsi la teneur juridique et socioéconomique du foncier.

- Les relations entre les droits de propriété et les droits d'usage : si a priori le propriétaire détient la majorité des droits d'usage sur ses parcelles, la mise en œuvre de l'action publique tend à reconnaître des droits d'usage à d'autres acteurs que le propriétaire (ici l'acteur public) ou à modifier la configuration des droits d'usage et de propriété. Dans ce sens, l'action publique déconstruit la relation entre droit de propriété et droit d'usage et fragilise ces derniers (avec par exemple la transformation de baux ruraux en conventions de prêts à usage).

$\mathrm{Ce}$ travail empirique, qui investit un champ de l'action publique environnementale, conforte donc les analyses antérieures qui avaient déjà mis en avant le rôle des acteurs privés (Commaille et Duran, 2009). On voit dans cette étude de cas combien les formes de contractualisation entre acteurs publics et privés deviennent la norme, les acteurs privés étant ici les garants de la bonne atteinte des objectifs des politiques publiques. On peut ici formuler l'hypothèse que les ressources (entre autres foncières, financières ou cognitives) dont ils disposent expliquent cette position privilégiée dans un contexte où celles des pouvoirs publics tendent à se restreindre, à tous les échelons. Surtout, on peut chercher à qualifier les modalités selon lesquelles de « groupe cible» ou destinataires de l'action publique, ces derniers tendent in fine à devenir des «facilitateurs » et une clé de voûte de sa mise en œuvre. 


\section{RÉFÉRENCES BIBLIOGRAPHIQUES}

Adam P., Debiais N., Malavoi J.-R. (2007). Manuel de restauration hydromorphologique des cours d'eau. Agence de l'eau Seine Normandie.

Allain S. (2004). La négociation comme concept analytique central d'une théorie de la régulation sociale. Négociations, De Boeck Université, 2004/2, n 2, p. 23-41.

Commaille J., Duran P. (2009). Pour une sociologie politique du droit. L'Année sociologique, Presses Universitaires de France, 2009/1, vol. 59.

Crozier M., Friedberg E. (1977). L'acteur et le système. Paris, Seuil.

Duran P., Thoenig J.-C. (1996). L'État et la gestion publique territoriale. Revue française de science politique, vol. 46, $\mathrm{n}^{\circ} 4$, p. 580-623.

Friedberg E. (1993). Le pouvoir et la règle : dynamiques de l'action organisée. Paris, Seuil.

Ghiotti S. (2007). Les territoires de l'eau. Gestion et développement en France. Paris, CNRS Éditions.
Knoepfel P., Kissling-Naf I., Varone F. (2001b). Régimes institutionnels de ressources naturelles : analyse comparée du sol, de l'eau et de la forêt. Bâle, Genève, Helbing \& Lichtenhahn.

Larrue C. (2000). Analyser les politiques publiques d'environnement. Paris, L'Harmattan.

Lascoumes P. (1990). Normes juridiques et mise en œuvre des politiques publiques. L'Année sociologique, $\mathrm{n}^{\circ} 40, \mathrm{p}$. 43-71.

Lascoumes P., Le Gales P. (2007). Sociologie de l'action publique. Paris, Armand Colin.

Syndicat Mixte Veyle Vivante (2004). Contrat de rivière Veyle.

Mény Y., Thoenig J.-C. (1989). Politiques publiques. Paris, PUF.

Thoenig J.-C. (1998). L'usage analytique du concept de régulation. Droit et Société, $n^{\circ} 24$, p. 35-53. 